\# Corresponding author: Peter Müllner, Department of Materials Science and Engineering, Boise State University, 1910 University Dr., Boise, ID 83725, USA; email: petermullner@boisestate.edu

\begin{abstract}
We coated the surfaces of Ni-Mn-Ga samples with hard TiN and soft elastomer and implanted $\mathrm{Nb}^{+}$ions. All of these treatments stabilized a dense twin structure. After removing the treated surface layers, the original coarse twin structure reappeared. Compression tests of the ionimplanted sample with fine twins exhibited a continuously increasing, smooth stress-strain curve. Without surface layer, the stress-strain curve was more rugged and flat, the twinning stress decreased, and one twin boundary moved throughout the entire sample.
\end{abstract}

Keywords: surface modifications, twinning, ferromagnetic shape memory 
Performance of magnetic shape memory (MSM) alloys strongly depends on the twin microstructure [1]. Homogeneous single crystalline Ni-Mn-Ga alloys with only one twin boundary exhibit twinning stresses as low as $0.05 \mathrm{MPa}$ [2]. Samples with a coarse twin structure are undesirable in many applications because they can kink extensively when strained and have a tendency to form intersecting twins which cause hardening $[3,4]$ and fracture $[5,6]$. Samples with a dense twin structure remain straight when they are strained and can sustain hundreds of millions of loading cycles [1,5]. Different twin boundary densities can be influenced via mechanical deformation and result in different twinning stresses, as show above $[3,7,8]$. Deformations in the surface layer of the Ni-Mn-Ga samples decrease twin width while increasing twinning stress $[9,10]$. This indicates that the surface defects generated by the deformations pin the twinning dislocations, which is in addition to internal defects as shown in thin Ni-Mn-Ga alloy foils [11].

The goal of this study was to stabilize a dense twin structure via surface treatments. We demonstrate that coatings and ion implantation of the surface increase the twin density and prevent them from coarsening during repeated straining.

We, first, studied the effect of a TiN coating on the stabilization of a dense twin structure on a $\mathrm{Ni}-\mathrm{Mn}-\mathrm{Ga}$ element, with dimensions of $1 \times 2.2 \times 20 \mathrm{~mm}^{3}$ (AdaptaMat Ltd., Finland). The sample had a $10 \mathrm{M}$ martensitic structure and the austenite start temperature was $44{ }^{\circ} \mathrm{C}$ with a twinning stress of 1.2 $\mathrm{MPa}$. The sample exhibited a two variant, coarse twin structure and strained about $6 \%$ in a sufficiently strong magnetic field. Three of the sample's long sides were coated, in its austenitic phase (i.e. at elevated temperature), with a $1 \mu \mathrm{m}$ layer of titanium nitride using physical vapor deposition, as indicated in the cross-sectional view of the sample shown in Fig. 1. The E-modulus of TiN thin films depend on deposition parameters and vary widely with reported valuesbetween 110 and $640 \mathrm{GPa}$ [12-14]. At room temperature, the sample exhibited an unordered, multi-variant twin structure typical for selfaccommodated martensite. This microstructure formed after cooling from the austenite due to the constraining surface stresses after coating the sample in its austenitic phase. An ordered, two-variant thin twin structure was created by turning a magnetic field of 1 Tesla several times between the directions of the long dimension $(20 \mathrm{~mm})$ and short dimension $(1 \mathrm{~mm})$. The length of the sample was measured with calipers before and after being exposed to the magnetic field. The sample strained about $1 \%$ in the magnetic field. With a ratio of film thickness to substrate thickness of $1 / 1000$ and taking into account that three sides of the crystal were coated, the stress in the film must be 500 times larger than the stress in the substrate and of opposite sign to counter balance the stress in the substrate. The magnetostress is about $3 \mathrm{MPa}$ [15]. This implies that the film stress did not exceed about 1.5 $\mathrm{GPa}$ at a strain of about $1 \%$, corresponding to an E-modulus of $150 \mathrm{GPa}$. This value is reasonable in the range of reported stiffness values. 1a shows the uncoated surface in the otherwise TiN-coated sample. Twin variants are seen as light and dark vertical stripes. The twins were very thin with an average width of only $15 \mu \mathrm{m}$ and remained even after straining the sample hundreds of times. The coating was removed by grinding and electropolishing 
which recovered the initial broad twin structure, as shown by the single twin boundary in Fig. $1 b$.

In a second study, a single crystal with the composition of $\mathrm{Ni}_{51.5} \mathrm{Mn}_{26.5} \mathrm{Ga}_{22}$ (in at\%) was grown and heat treated as described in Ref. [16] and a sample of $1 \times 2 \times 20 \mathrm{~mm}^{3}$ was cut from the single crystalline bar. The austenite start temperature of the alloy was $32{ }^{\circ} \mathrm{C}$. The twinning stress for the crystal containing one twin boundary was $0.18 \mathrm{MPa}$. The sample was coated with a transparent elastomeric resin (Dow Corning, Sylgard 184 Silicone Elastomer by casting the resin over the Ni-Mn-Ga rod when the rod was fully compressed. The E-modulus of this Elastomer depends on casting and hardening parameters and reported values vary between 1.8 $\mathrm{MPa}$ [17] and 2.6 MPa [18] depending on hardening. The sample remained martensitic while the elastomer cured due to its low curing temperature. After curing, the elastomer was cut so that the thickness of the elastomeric layer around the sample was $1 \mathrm{~mm}$. With the very low E-modulus, the elastomer provides virtually zero restoring force. Fig. 2 shows the twin structure and a cross-sectional view of the sample after exposure to a magnetic field. The twins were thin (about $60 \mu \mathrm{m}$ ), regularly spaced and were present even after the sample was strained. These twins were coarser than those in the TiN-coated sample. The difference in twin widths is likely due to the elastomeric resin's lower stiffness as compared to that of TiN, with the E-modulus of TiN more than two orders of magnitude higher than of elastomer coating. The initially coarse twin structure was recovered after the elastomeric resin was removed by acetone, as shown in Fig. 2b. A single twin boundary and two twin variants (light and dark areas) are seen in Fig. $2 b$.

In the third part of this study, twin microstructures and twinning stresses were investigated on an ion implanted single crystalline Ni-Mn-Ga sample (AdaptaMat Ltd., Finland) with dimensions of $0.9 \times 2.4 \times 20 \mathrm{~mm}^{3}$. The sample exhibited a $10 \mathrm{M}$ martensitic structure with an austenite start temperature of $44{ }^{\circ} \mathrm{C}$. The surfaces of the sample were ground and electropolished. The sample was bent to create a dense twin structure, as shown in Ref. [2]. Ion implantation was performed on the sample for two hours at liquid nitrogen temperature using $\mathrm{Nb}^{+}$ions at a voltage of $400 \mathrm{kV}$ and a fluence of $2 \times 10^{15}$ ions $/ \mathrm{cm}^{2}$. The deformed surface layer was about $100 \mathrm{~nm}$ thick [19]. After ion implantation, an $8.2 \mathrm{~mm}$ long sample was cut from the ion implanted sample for mechanical testing in compression. After a first series of compression tests, the ion implanted sample was then electropolished, using 30\% nitric acid in ethanol solution, three times with $20 \mathrm{~V}$ for 15 seconds each time. After each electropolishing step, compression tests were performed.

The twinning stress and the change of the twin microstructure of the ion implanted sample was determined by using a modified Zwick 1445 mechanical test bench (Zwick, Ulm, see [20] for experimental details) to compress the sample parallel to its longest dimension at a constant cross-head speed of $0.25 \mathrm{~mm} / \mathrm{min}$. Before each compression test, the sample was manually elongated parallel to the longest dimension of the sample to prepare the sample for the next compression test. The surface of the sample was video-recorded in-situ during the deformation tests with an Infinity-X camera (Lumera Scientific Inc.) so that the motion of the twin boundaries could be seen. The sample was mechanically tested four times with the ion 
implanted surface. Fig. 3 shows the stress-strain curves of the ion implanted sample. The curves were smooth and continuously increasing. The average twinning stress was about 1.5 $\mathrm{MPa}$. The video of the compression test showed fine vertical twins, $40 \mu \mathrm{m}$ in width, covering the entire length of the sample.

The compression test results of the electropolished sample are presented in Fig. 4 for four subsequent electropolishing steps. The stress-strain curves are serrated and have decreased slopes compared to the ion implanted sample. The slope of the stress-strain curves decreased after each electropolishing. Similarly, the average twinning stress decreased after each electropolishing step and the curve was serrated in contrast to the smooth curve in Fig. 3 (ion implanted sample). Curve 4, epol 3 in Fig. 4 represents the state after complete removal of the ion implanted surface layer. This curve showed a plateau-like behavior, and a twinning stress of about $0.7 \mathrm{MPa}$. The video of the electropolished sample revealed only one twin boundary producing a macroscopic kink moving through the sample. The fine twin structure had disappeared completely.

In the coated samples (both with titanium nitride as well as with the elastomer coating) twins were thin. The experiments demonstrate that the coatings stabilized the dense twin structure and acted as surface constraints in a similar way as mechanical constraints [1] or surface deformations [9] do. The coatings also prevented twins from coarsening and kept the twin structure fine even after straining the sample a large number of cycles. The TiN coating with a higher stiffness created a denser twin structure than the elastomeric coatings, although the coating layer was much thinner $(1 \mu \mathrm{m})$ than that of the elastomer $(1 \mathrm{~mm})$. The thin twin structure was stabilized by coating only three sides of the sample with TiN.

The twin microstructure of the ion implanted sample had thin twins across the entire sample, similar to the twin microstructures of samples whose surfaces were mechanically deformed [9]. Ion implanted crystals had smooth stress-strain curves with relatively steep slopes. This mechanical behavior is again similar to that of crystals with deformed surfaces [9]. Similar to mechanically-induced surface defects, ion implantation increased the twinning stress and resulted in continuously increasing, smooth stress-strain curves. When the surface layer (ion implanted or deformed) was removed by electropolishing, the sample's mechanical behavior and twin structure became similar to single crystals without surface treatments: the twinning stress was low, the slope of the stress-strain curve was small (often plateau-like), and the curve was serrated [2]. We conclude that ion implantation creates lattice imperfections or a dead layer with a different, hard phase than the bulk material which pin the twinning dislocations similarly to mechanically-induced surface deformation.

It has been shown that the ceramic TiN and elastomeric surface coatings as well as ion implantation stabilize a dense twin microstructure and prevent twins from coarsening during repeated straining for many cycles. Ion implantation also creates smooth, continuously increasing stress-strain curves and increases the twinning stress. When the coated or ion implanted surface layers are removed, a coarse twin structure reappears and the twinning 
stress decreases to a level typical of homogeneous single crystalline samples. Local defects produced by the surface treatments pin the twinning dislocations and stabilize the dense twin structure.

\section{Acknowledgements}

We thank Phil Boysen for machining a sample holder for this study and Aaron Smith for reviewing and discussing the manuscript. We acknowledge the U.S. Department of Energy, Office of Basic Energy Science, Division of Materials Sciences and Engineering under Award No. DEFG-02-07ER46396 for supporting all experimental work. MC thanks the Deutsche Forschungsgemeinschaft (Grant No. SPP1239 Schn 1106/1) for partial financial support. PM is grateful to ETH Zürich for donating magneto-mechanical testing devices. 


\section{References}

[1] M. Chmielus, V.A. Chernenko, W.B. Knowlton, G. Kostorz, P. Müllner, Eur. Phys. J. Sp. Top. 158 (2008) 79.

[2] L. Straka, N. Lanska, K. Ullakko, A. Sozinov, Appl. Phys. Lett. 96 (2010) 131903.

[3] L. Straka, H. Hänninen, N. Lanska, A. Sozinov, J. Appl. Phys. 109 (2011) 063504.

[4] V. Pinneker R. Yin, C. Eberl, A. Sozinov, Y. Ezer, M. Kohl, J. Alloys Comp. 577 (2013) S358.

[5] P. Müllner, V.A. Chernenko, G. Kostorz, MRS Symp. Proc. 785 (Warrendal, PA, 2004), p. 415.

[6] I. Aaltio, A. Soroka, Y. Ge, O. Söderberg, S.-P. Hannula, Smart. Mater. Struct. 19 (2010) 075014.

[7] I. Aaltio, O. Söderberg, Y. Ge, S.-P. Hannula, Scripta Mater. 62 (2010) 9.

[8] L. Straka H. Hänninen, A. Soroka, A. Sozinov, J. Phys. Conf. Series 303 (2011) 012079.

[9] M. Chmielus, K. Rolfs, R. Wimpory, W. Reimers, P. Müllner, R. Schneider, Acta Mater. 58 (2010) 3952.

[10] M. Chmielus, C. Witherspoon, K. Ullakko, P. Müllner, R. Schneider, Acta Mater. 59 (2011) 2948.

[11] O. Heczko, A. Soroka, S.-P. Hannula, Appl. Phys. Lett. 93 (2008) 022503.

[12] S.H. Kim, H. Park, K.H. Lee, S.H. Jee, D.J. Kim, Y.S. Yoon, H.B. Chae, J. Ceramic. Proc. Res. 10 (2009) 49;

[13] D.S. Stone, K.B. Yoder, W.D. Sproul, J. Vac. Sc. Techn. A 9 (1991) 2543;

[14] A. Karimi O. R. Shojaei, T. Kruml, J. L. Martin, Thin Solid Films 308 (1997) 334.

[15] P. Müllner, V. A. Chernenko, M. Wollgarten, G. Kostorz, J. Appl. Phys. 92 (2002) 6708.

[16] D. Kellis, A. Smith, K. Ullakko, P. Müllner, J. Crystal Growth 359 (2012) 64.

[17] K. M. Choi, J. A. Rogers, J. Am. Chem. Soc. 125 (2003) 4060.

[18] F. Schneider, T. Fellner, J. Wilde, U. Wallrabe, J. Micromech. Microeng. 18 (2008) 065008

[19] O. Toader, University of Michigan Ion Beam Laboratory, Private communication

[20] P. Müllner, V.A. Chernenko, G. Kostorz. Scr. Mater. 49 (2003) 129. 


\section{Figure Captions}

Figure 1. (a) After more than 100 magnetic cycles, thin twins (vertical stripes) are seen on the non-coated surface of a Ni-Mn-Ga sample of which three sides have been coated with TiN $(1 \mu \mathrm{m})$, as illustrated in the cross-sectional view $A$-A. (b) Two twin variants (dark and light areas), separated by a single twin boundary, reappear after the TiN coating was removed by grinding and electropolishing. The cross sectional view $B-B$ is shown on the right.

Figure 2. (a) Thin twins (vertical stripes) of a Ni-Mn-Ga sample that was coated on all sides with a transparent elastomeric layer $(1 \mathrm{~mm})$, as illustrated in the cross-sectional view $A-A$. (b) Two twin variants (dark and light areas), separated by a single twin boundary, reappear after removing the elastomeric coating by acetone. The cross sectional view $B-B$ is shown on the right.

Figure 3. Stress-strain curves of four compressive deformation tests of the ion implanted NiMn-Ga sample. Between each deformation test, the sample was manually elongated parallel to its longest dimension.

Figure 4 (color online). Stress-strain curves of four compressive deformation tests of the ion implanted and progressively electropolished Ni-Mn-Ga sample. The labels indicate which deformation cycle is shown and how often the sample was electropolished (epol 1 for one electropolishing, epol 2 for two and so on). Between each compressive deformation and electropolishing, the sample was manually elongated parallel to its longest dimension. 


\title{
Stabilizing a fine twin structure in Ni-Mn-Ga samples by coatings and ion implantation
}

\author{
K. Ullakko ${ }^{1}$, M. Chmielus ${ }^{2}$ and P. Müllner ${ }^{3 \#}$ \\ ${ }^{1}$ Lappeenranta University of Technology, LUT Savo Sustainable Technologies, Savonlinna, Finland \\ ${ }^{2}$ Department of Mechanical Engineering and Materials Science, University of Pittsburgh, Pittsburgh, \\ PA, USA \\ ${ }^{3}$ Department of Materials Science and Engineering, Boise State University, Boise, ID, USA \\ petermullner@boisestate.edu.
}

\section{Figures}

Figure $1-2$ columns wide

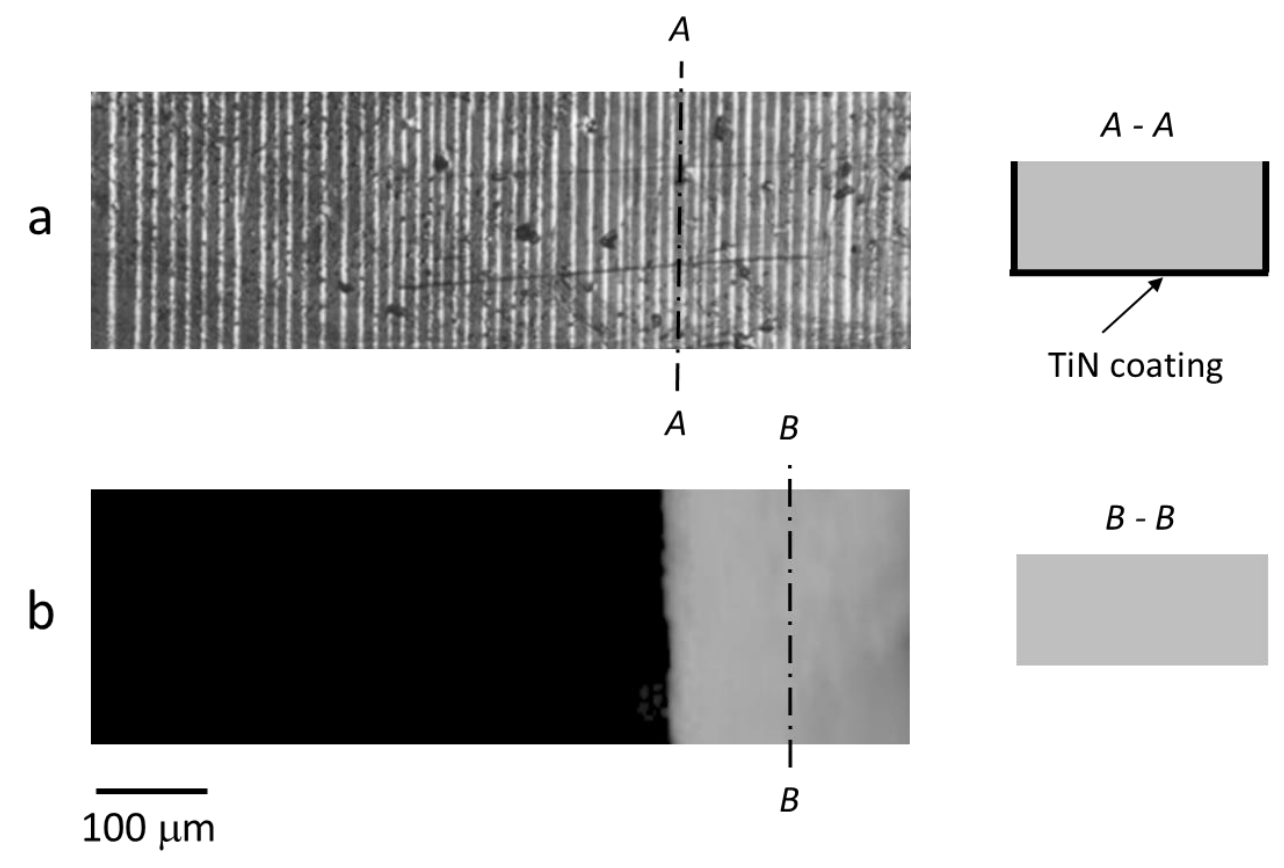

\# Corresponding author: Peter Müllner, Department of Materials Science and Engineering, Boise State University, 1910 University Dr., Boise, ID 83725, USA; email: petermullner@boisestate.edu 
Figure $2-2$ columns wide

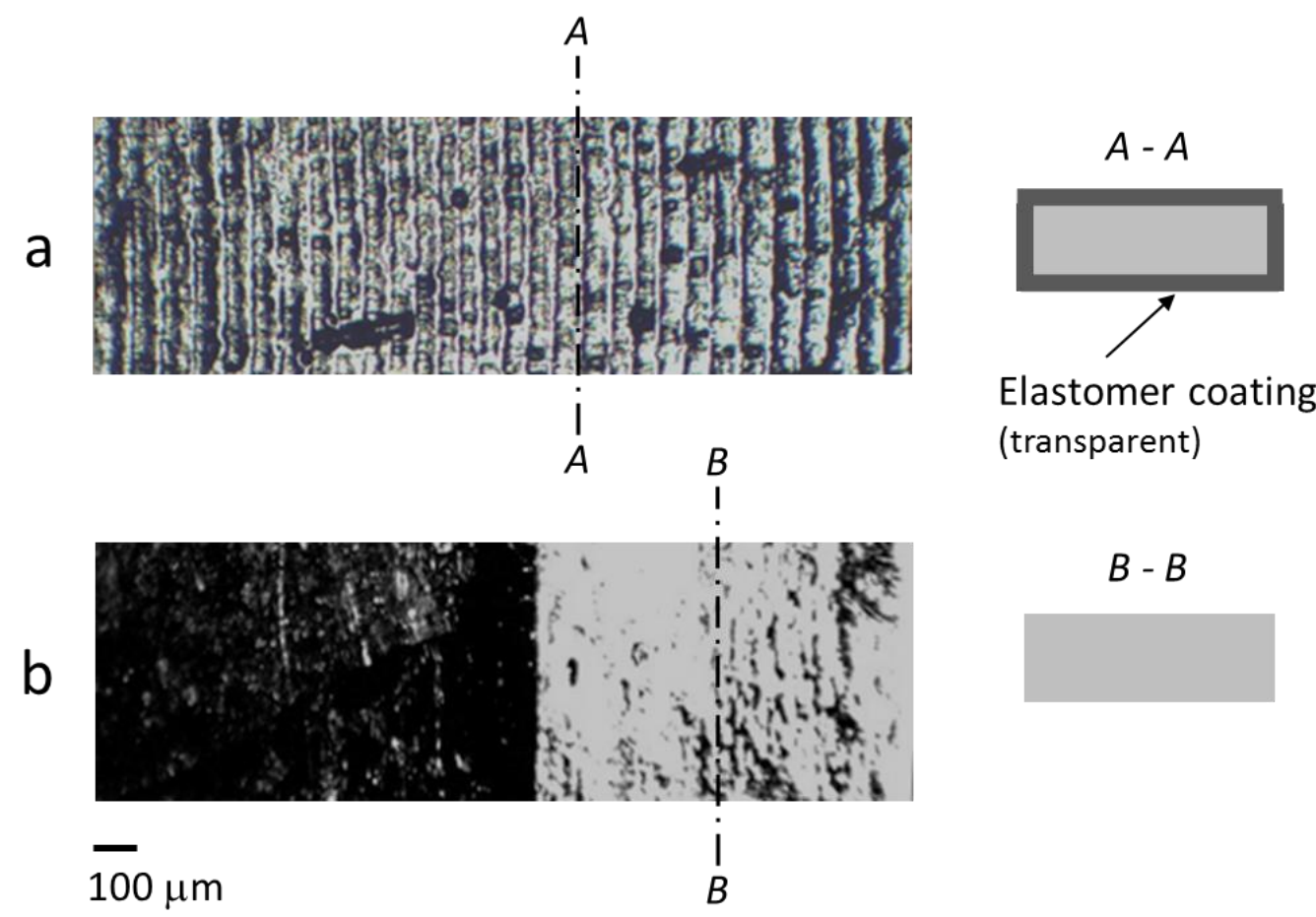


Figure 3 - 1 column wide

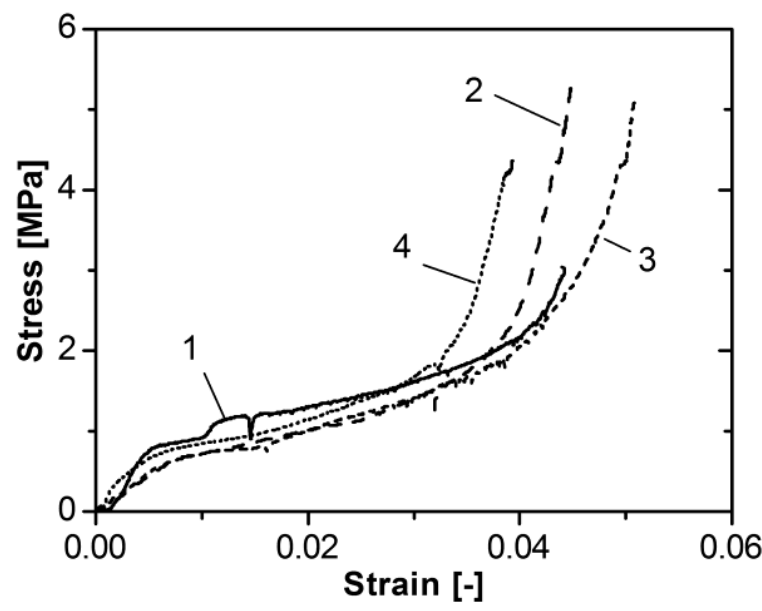


Figure 4 - 1 column wide - color online
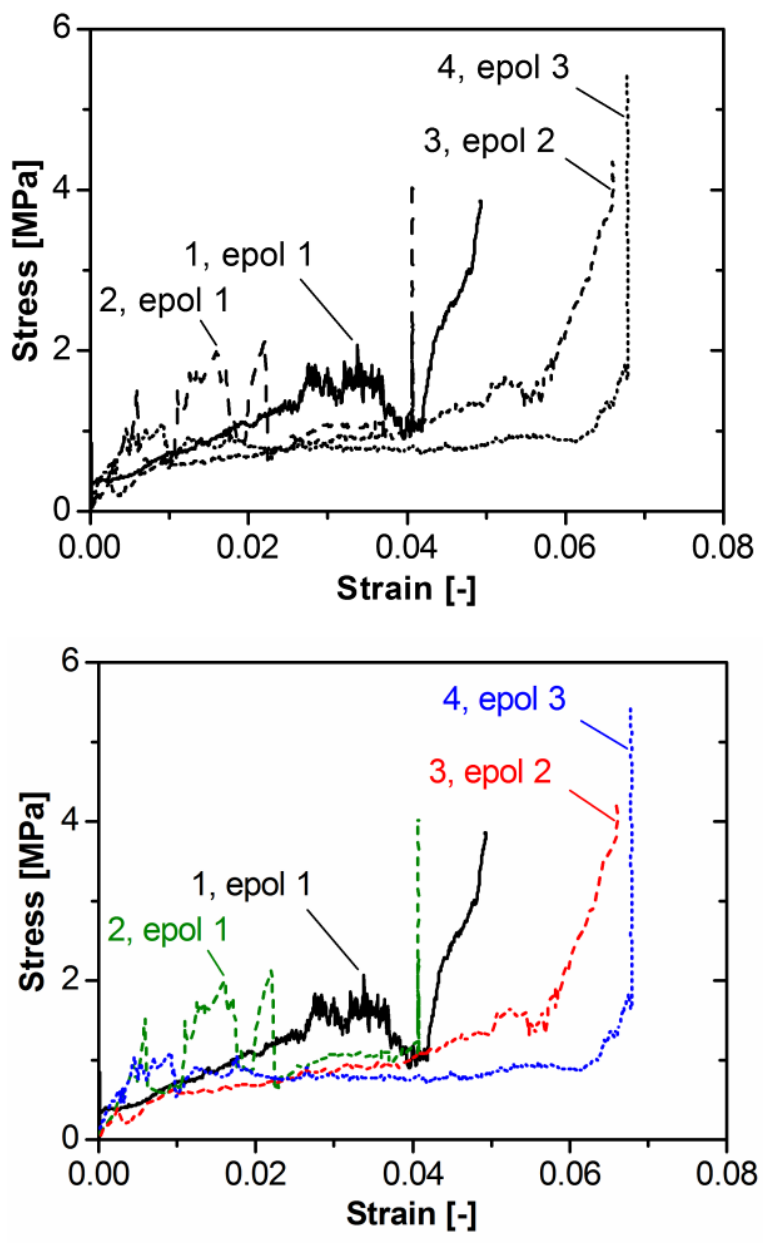